\title{
V INHALTSVERZEICHNIS
}

Für den inhait der einzelnen corpusbände - die sammlung musste sich in anbetracht des reichen materials entgegen dem ursprünglichen plan vornehmlich auf diese beschränken - verweise ich auf das literaturverzeichnis in den 'Altlateinischen inschriften's. 55 ff.

\begin{tabular}{|c|c|c|c|c|c|c|}
\hline CIL II & & CIL II & & CIL III & & CIL III \\
\hline $3^{8}$ & I 432 & $5 \circ 35$ & 723 & 1895 & 234 & 2880 \\
\hline 89 & I 141 & 5044 & 392 & 1903 & 1521 & $3107 \mathrm{a}$ \\
\hline 376 & 61 & 5144 & 42 & 1922 & 849 & $3174 b$ \\
\hline 405 & I 245 & 5227 & 501 & I 93 I & 254 & 3182 \\
\hline 425 & 848 & 5265 & $108 x$ & 1966 & 90 & $338 \mathrm{I}$ \\
\hline 488 & $3^{85}$ & 5342 & 243 & 2007 & 239 & 3399 \\
\hline 554 & 588 & 5393 & 922 & 2008 & I34 & 3402 \\
\hline $95^{2}$ & I 497 & 5416 & 789 & 2044 & 940 & 3447 \\
\hline IOI9 & 357 & 5535 & 306 & 2063 & 597 & 3474 \\
\hline I I IO & 599 & 5698 & $4^{68}$ & 2098 & I I $5^{\circ}$ & 3493 \\
\hline I 126 & 88 & 5713 & 447 & $2 \mathrm{II} 2 \mathrm{f}$ & I5 50 & 3498 \\
\hline 1222 & $65^{8}$ & 5720 & 1044 & 2126 & 702 & 3499 \\
\hline 1228 & 764 & 5728 & 1172 & $213^{\circ}$ & 28 & 3620 \\
\hline 1240 & 700 & $574^{2}$ & I 44 & 2147 & I I 6 & $3^{800}$ \\
\hline 1329 & 320 & $5^{821}$ & $3^{80}$ & 2156 & 817 & 39 I 4 \\
\hline I 8 I 8 & 692 & 5910 & 698 & 2162 & 305 & 4185 \\
\hline 1876 & 790 & 6153 & $5^{24}$ & 2225 & $54^{\circ}$ & 4282 \\
\hline 2071 & 1463 & 6265 & 746 & 2234 & 504 & $43 I 4$ \\
\hline 2094 & 300 & $6267 a$ & I 282 & 2240 & 1135 & $444 \mathrm{I}$ \\
\hline 2679 & 570 & 6299 & I I I 6 & 2263 & 953 & 4466 \\
\hline 2996 & 951 & 6302 & 89 & 2267 & 1179 & 4480 \\
\hline 2997 & I 59 & p. 1025 & 879 & 2284 & 621 & 4570 \\
\hline 3015 & 26 & CIL III & & 2296 & 666 & 4603 \\
\hline 3125 & 5 & 44 & 937 & 2326 & 95 & 4766 \\
\hline 3214 & 225 & 70 & I2 I9 & 2348 & 571 & 4796 \\
\hline 3244 & $49 I$ & 79 & I38Ia & $237^{\circ}$ & 682 & $4850 a$ \\
\hline 3347 & 699 & 108 & I 29 I & 2377 & $113^{8}$ & 4908 \\
\hline 3565 & 620 & 188 & 437 & 2406 & 469 & 5061 \\
\hline 3670 & 515 & $37 \mathrm{I}$ & 1040 & 2427 & 589 & 5277 \\
\hline 3762 & $3^{65}$ & 387 & 793 & 2434 & 972 & 5295 \\
\hline $4 I 73$ & 715 & $3^{87}$ & 812 & 2467 & 593 & 5482 \\
\hline 4174 & 1016 & 410 & 1039 & 2564 & 833 & 5622 \\
\hline 4291 & 275 & 917 & 568 & 2567 & $94^{8}$ & 5839 \\
\hline $433 I$ & I I $6 \mathrm{I}$ & 925 & 757 & 2583 & 932 & 5967 \\
\hline 4332 & 530 & $98 \mathrm{I}$ & 537 & 2602 & IO9I & 5968, I37 \\
\hline 4386 & 753 & $104 \mathrm{I}$ & I 286 & 2627 & 495 & 6010 \\
\hline 4405 & I 20 & I I94 & 691 & 2696 & 271 & 6122 \\
\hline 4442 & I95 & 1537 & 1283 & 2702 & 288 & 6137 \\
\hline 4512 & I 45 & 1738 & 1489 & 2811 & 478 & 6147 \\
\hline 4963 & 657 & 1743 & I $34^{8}$ & 2817 & I37 & 6380 \\
\hline
\end{tabular}


$\mathrm{v}$ inhaltsverzeichnis

169

\begin{tabular}{|c|c|c|c|c|c|c|c|}
\hline CIL & & CIL III & & CII III & & CIL V & \\
\hline $644 \mathrm{I}$ & $134^{\circ}$ & 9735 & 109 & 13374 & I392 & 896 & 86 \\
\hline 6598 & 4 & 9973 & 1473 & 13406 & 374 & $9 I_{4}$ & $34^{8}$ \\
\hline $66 \mathrm{II}$ & 294 & I01 95,1 & 583 & 13663 & I 276 & 1039 & 907 \\
\hline 6717 & 728 & 10202 & 1464 & 13917 & 1153 & II 52 & $93 I$ \\
\hline 6761 & 14 & I0289 & 295 & 14014 & 155 & 1870 & 559 \\
\hline 6801 & 256 & I0393 & 69 & 14062 & I 24 & 2007 & I 126 \\
\hline 6891 & 158 & 10453 & I I 7 I & I 4107 & 720 & 2013 & 409 \\
\hline 7058 & 1457 & 10457 & 586 & $14 I^{1} 4, I 3$ & IO74 & $2039 \mathrm{a} / \mathrm{b}$ & 783 \\
\hline $714^{8}$ & I I 54 & I0495 & I 6 I & $14147^{6}$ & I38 Ia & 2046 & 253 \\
\hline 7291 & 818 & 10518 & 687 & $14156^{2}$ & 318 & 2187 & 410 \\
\hline 7475 & $8 I_{4}$ & 10783 & I I I 7 & 14190 & I394 & 2313 & \\
\hline 7521 & 1054 & 10833 & 289 & $14206^{21}$ & 915 & $245^{2}$ & I IO3 \\
\hline 7535 & 1053 & I0837 & 946 & $1_{4} 2 \mathrm{I} 3$ & 1285 & 2701 & 1090 \\
\hline 7547 & 1079 & I I054 & 230 & $14214^{20}$ & 1302 & 2884 & 497 \\
\hline 7553 & 226 & I 1076 & 285 & 14237 & 1322 & $295^{6}$ & 921 \\
\hline & 271 & I 1082 & $1464 \mathrm{~b}$ & $14^{2} 4^{1}$ & 346 & $30 \mathrm{I}_{4}$ & 1028 \\
\hline 7681 & 334 & I 1098 & 774 & 14269 & 727 & 3034 & 998 \\
\hline 7693 & 153 & I I I I 8 & 231 & $I_{4275}^{4}$ & 815 & 3259 & 33 \\
\hline 7756 & I 204 & I 1240 & $48 I$ & 14292 & 1537 & $333^{2}$ & 580 \\
\hline 7791 & I 354 & I I 308 & 370 & $143^{2} I^{26}$ & roo4 & 3496 & I 4 I I \\
\hline 7827 & $\begin{array}{l}3038 \\
103\end{array}$ & I I 45 I & 792 & $143^{22}{ }^{13}$ & I 84 & 3516 & 238 \\
\hline 7856 & $8 \mathrm{I} 3$ & I 1634 & 914 & $1434^{8}$ & 1353 & 3555 & 784 \\
\hline 8029 & I349 & I 1697 & $133^{8}$ & I 4353 & 198 & 3634 & 304 \\
\hline 8043 & 617 & I 1700 & 257 & I $4354^{16}$ & $922 \mathrm{a}$ & 3653 & 1469 \\
\hline 8324 & 835 & I 1926 & 165 & $I_{4359^{20}}$ & $153^{8}$ & 3683 & 595 \\
\hline 8403 & 1489 & I 1977 & 258 & $14360^{20}$ & 1200 & 3772 & 473 \\
\hline 8412 & 123 & I 2014,38 I & $46 r$ & $143^{6} 7^{1}$ & $105^{\circ}$ & 3801 & 39 \\
\hline 8447 & 151 & I 2054 & 529 & $143^{84}$ & I I 89 & 3996 & 1013 \\
\hline 8500 & 686 & I 207 I & 323 & $1_{4406} \mathrm{a}$ & I 128 & 4104 & 969 \\
\hline 8509 & 1190 & 12377 & I 535 & $14433^{1}$ & 503 & 4220 & 750 \\
\hline 8591 & 1510 & 12392 & I 234 & $14503^{1}$ & 255 & $44 I 0$ & 610 \\
\hline 8729 & 453 & I 2396 & 564 & 14524 & I 54 & 4445 & 499 \\
\hline 8729 & 135 & 12422 & I 389 & I $4599^{1}$ & $43^{8}$ & 4488 & 785 \\
\hline 8742 & 480 & I 2433 & 669 & $14599^{2}$ & I I 93 & 4489 & I 262 \\
\hline 8820 & 573 & I 2437 & 224 & 14809 & I 186 & 4510 & 899 \\
\hline $883^{\circ}$ & 278 & I 2466 & 1265 & 14855 & 667 & 4525 & 706 \\
\hline 8831 & 1393 & 12476 & 1076 & $14943^{1}$ & 794 & 4612 & 1460 \\
\hline 8862 & 5 II & 12484 & 1203 & 14977 & 293 & $46 I 6$ & $5^{8}$ \\
\hline 8876 & $9 I$ & I 2489 & $26 \mathrm{I}$ & 15035 & 997 & 4721 & 328 \\
\hline 8910 & 1094 & 12495 & I5 & 15055 & 844 & 4754 & 611 \\
\hline 8935 & 298 & 12558 & 30 & 15131 & 572 & 4942 & 773 \\
\hline $893^{8}$ & 574 & 12598 & 1073 & I 5166 & I I & 5134 & 531 \\
\hline 8951 & 979 & 12602 & $45 \mathrm{I}$ & $15205 a$ & $5^{18}$ & 5157 & 206 \\
\hline 8971 & 680 & I 2680 & 719 & $15216^{11}$ & 725 & 5337 & I 496 \\
\hline 9009 & 506 & 12711 & 8 & 15219 & 880 & $55 \mathrm{IO}$ & 474 \\
\hline 9029 & 592 & I 2799 & 924 & CIL V & & 5583 & 842 \\
\hline 9178 & 567 & I 2894 & 482 & $2 I$ & 886 & $566 \mathrm{I} a$ & I 279 \\
\hline 9228 & I 199 & I 2949 & 736 & 128 & 607 & 5902 & I 534 \\
\hline 923 & 164 & I 2953 & 1043 & 129 & 625 & 5931 & 34 \\
\hline 93 & 1319 & 13013 & 970 & 222 & 1516 & 6061 & 517 \\
\hline 9364 & 324 & 13037 & 971 & 893 & 1482 & 6372 & 85 \\
\hline $945^{\circ}$ & 63 & I 3079 & $4 I_{4}$ & 895 & $1464 a$ & 6547 & 612 \\
\hline
\end{tabular}




\begin{tabular}{|c|c|c|c|c|c|c|c|}
\hline CIL, V & & CIL V & & CIL VI & & CIL VI & \\
\hline 6580 & 712 & 3714 & I 182 & 5817 & 640 & $85^{82}$ & 393 \\
\hline 6615 & I 26 & $3722 \mathrm{a}$ & 1070 & 5822 & I & 8606 & 1412 \\
\hline 6710 & 1031 & 3941 & 949 & $595 I$ & 210 & 8670 & 32 \\
\hline 7464 & II 73 & 3992 & 1310 & 5982 & 1289 & 8688 & 1294 \\
\hline 7465 & 105 & 4087 & 902 & $599 \mathrm{I}$ & 47 I & 8761 & 465 \\
\hline 7537 & I 176 & 4118 & 935 & 6156 & 1523 & 8766 & 167 \\
\hline 7615 & 1263 & 4155 & 767 & 6182 & 1097 & 8865 & 584 \\
\hline 7647 & 377 & 4174 & 901 & 6213 & I3 I I & 8875 & ro75 \\
\hline 8131 & 609 & 4222 & 1270 & 6216 & 1409 & 8885 & 1386 \\
\hline 8136 & 484 & 4265 & 639 & 6242 & 693 & 8937 & 1225 \\
\hline 8138 & 608 & 4315 & 146 & 6282 & 349 & 8983 & 910 \\
\hline 8254 & 435 & 4355 & 1.226 & 6326 & 895 & 8985 & 1224 \\
\hline 8273 & I 539 & 4356 & 704 & 6469 & I509 & 8991 & 1381 \\
\hline 8485 & 361 & 4407 & 367 & 6492 & IOO & 8999 & 1297 \\
\hline CIL VI & & 4410 & I 49 I & 6524 & 247 & 9232 & 1253 \\
\hline 68 & $133^{\circ}$ & 4416 & 263 & 6555 & 131 & $925^{8}$ & 1228 \\
\hline 303 & 1532 & 44 I9 a & $44^{\circ}$ & 6565 & 894 & 9408 & 13 I 2 \\
\hline 377 & 777 & 4428 & 721 & 6571 & 775 & 9422 & 1443 \\
\hline 406 & 1554 & 4432 a & 577 & 6639 & 152 & 9477 & 422 \\
\hline $46 r$ & 1278 & $4444 a$ & $3^{10}$ & 6792 & 218 & 9592 & 766 \\
\hline 730 & I 237 & 4457 & 193 & $68 \mathrm{I} 4$ & I 308 & 9604 & 1106 \\
\hline 735 & 1341 & 4477 & I 293 & 6870 & 180 & 9659 & I 5 I I \\
\hline $746 \mathrm{~b}$ & I029 & 4516 & I 28 & 7017 & 799 & 9663 & 1026 \\
\hline 880 & 1284 & 4521 & 925 & 7093 & 411 & 9792 & 1272 \\
\hline I 490 & $132 \mathrm{I}$ & $453^{\circ}$ & 1519 & 7260 & 1162 & 10074 & 133 \\
\hline 1537 & 1229 & $453^{2}$ & 421 & $7281 \mathrm{a}$ & 999 & IOI 85 & 821 \\
\hline 2120 & I 553 & $455^{\circ}$ & 35 & 7297 & 1222 & IOI 86 & $\mathrm{I}_{3} \mathrm{I}_{4}$ \\
\hline 2273 & 1239 & 4565 & $95^{8}$ & 7308 & I 129 & 10238 & 837 \\
\hline 2464 & $55 \mathrm{I}$ & 46 I a & 770 & 7318 & 315 & I0246 & I9 \\
\hline $254^{8}$ & 1368 & 4625 & 88 I & 7375 & I 44 I & 10268 & 942 \\
\hline 2570 & $34^{\circ}$ & 4802 & I 42 I & $7457 \mathrm{~b}$ & I 254 & 10273 & 249 \\
\hline 2601 & I 4 I & 4870 & 1022 & $745^{8}$ & 1207 & 10276 & 1195 \\
\hline 2662 & 1088 & 4887 & 264 & 7470 & I I 94 & 10322 & 1350 \\
\hline 2759 & 1465 & 4960 & 390 & 7516 & 80 & 10329 & 1258 \\
\hline 2774 & 888 & 4966 & 1257 & 7577 & $14^{20}$ & 10346 & 1367 \\
\hline $284 \mathrm{I}$ & I 466 & 4999 & 526 & 7612 & 1223 & 10458 & 73 \\
\hline 2907 & $135^{8}$ & 5028 & 200 & 7631 & 893 & I0464 & 188 \\
\hline 2963 & $123^{\circ}$ & 5070 & 916 & $765^{\circ}$ & 641 & IO554 & 204 \\
\hline 3198 & 1298 & 5108 & 321 & $765^{2}$ & I 295 & 10703 & 509 \\
\hline 3282 & 248 & 5174 & 624 & $776 \mathrm{I}$ & 443 & 10718 & 472 \\
\hline 3293 & 994 & 5175 & r 439 & 7770 & 1474 & ro73I & 632 \\
\hline 3446 & 820 & 5176 & 889 & 7853 & I 399 & 10791 & IOI9 \\
\hline 3452 & 642 & $5187 b$ & I 25 & $795^{8}$ & 890 & 10902 & 538 \\
\hline 3454 & 1437 & $5206 a$ & 1363 & 7974 & 212 & 10939 & 865 \\
\hline 3478 & 187 & $534^{\circ}$ & 78 & 7990 & 796 & 10944 & 507 \\
\hline 3490 & I 404 & 5375 & $35^{2}$ & 80 I a & I I IO & I I 232 & 48 \\
\hline 3557 & I I 2 I & $544^{8}$ & 496 & 8189 & $96 I$ & I I 49 I b & 1316 \\
\hline 3566 & 70 & $555^{2}$ & 934 & 8207 & 130 & I 1645 & 74 \\
\hline 3604 & 1379 & $55^{8} 8$ & I 22 I & 8247 & 864 & I I 69I & 291 \\
\hline 3608 & 643 & 5601 & 1071 & 8492 & 882 & I I 77 I & 622 \\
\hline 3631 & 729 & 5639 & 795 & 8523 & 797 & I I 993 & 866 \\
\hline 3637 & 759 & 5662 & 97 & 8539 & ro69 & II 939 & 1355 \\
\hline 3711 & 98 & 5714 & 941 & $858 \mathrm{I}$ & 673 & I 2006 & 867 \\
\hline
\end{tabular}




\begin{tabular}{|c|c|c|c|c|c|c|c|}
\hline CIL VI & & CIL VI & & CIL VI & & CIL VI & \\
\hline 12055 & 1453 & r6809 & 425 & 21322 & $\mathrm{I} 46 \mathrm{I}$ & 25961 & I I 3 I \\
\hline I $2116 / 7$ & 550 & I 7034 & 459 & $2154^{8}$ & IO2 & $26010 a$ & 219 \\
\hline 12375 & 883 & 17072 & 1504 & 2 I $800 \mathrm{a}$ & 387 & 26042 & 987 \\
\hline I 2405 & 170 & 17203 & 235 & 22120 & IOI 8 & 26 I I 5 & 803 \\
\hline $12607 / 8$ & 1514 & I7297 & 1547 & 22163 & 72 & 26II9 & I 503 \\
\hline I 2675 & 1066 & I 7330 & 1325 & 22400 & $65 I$ & 26121 & 1423 \\
\hline 12737 & $\log 2$ & I 7349 & 582 & 22437 & $3^{6}$ & 26132 & $3^{89}$ \\
\hline 13070 & 237 & I 7387 & 65 & 22520 & 871 & 26133 & 605 \\
\hline 13075 & 1303 & I 7408 & 1499 & 22647 & 1068 & 26158 & 1384 \\
\hline 13080 & 1055 & 17469 & $37 I$ & 23176 & 1220 & 26227 & 1356 \\
\hline 13099 & 1227 & I7508 & 527 & 23334 & 646 & 26274 & 976 \\
\hline 13101 & I380 & I 7565 & 1545 & $2355^{2}$ & 1082 & 2655 I & 1442 \\
\hline 13102 & 401 & 17622 & 1388 & 23606 & 250 & 26584 & 492 \\
\hline 13 I 4 & 280 & 17633 & IO4I & 23629 & 1024 & 26601 & 600 \\
\hline 13146 & 1400 & 17677 & 1498 & 23660 & I I I 2 & 26680 & 1166 \\
\hline 13150 & 423 & 17690 & 816 & 23680 & 1327 & 26688 & 996 \\
\hline 13170 & 581 & 17803 & 189 & 23774 & 1323 & 26704 & I 543 \\
\hline $13 \times 76 / 7$ & 1051 & 17987 & 441 & 24339 & 917 & 26708 & 232 \\
\hline 13246 & 606 & I 8086 & $63 I$ & $2435^{\circ}$ & 1099 & 26713 & $75^{8}$ \\
\hline 13271 & I 242 & I8107b & $126 \mathrm{I}$ & 24471 & 647 & 26849 & 1065 \\
\hline 13292 & 4I 2 & 18 I 23 & 644 & 24475 & 872 & 26850 & 1513 \\
\hline 13302 & 316 & 18327 & 486 & 24571 & 187 & 26942 & 46 \\
\hline 13364 & 601 & 18532 & 228 & $24724 a$ & 378 & 27009 & I I I \\
\hline $133^{8} 3$ & 936 & $1853^{6}$ & 245 & 24724 a & 827 & $270_{4} I$ & I 188 \\
\hline I3394 & I33 I & 18659 & 1480 & 24811 & 918 & 27109 & I306 \\
\hline I3574 & I 40 & 18744 & 525 & 24972 a & 20 & 27136 & 769 \\
\hline 13714 & I 86 & $1875^{8}$ & I 209 & 25000 & 405 & 27196 & I 496 \\
\hline I 3830 & 1422 & 18773 & 399 & 25017 & $73 I$ & 27259 & 986 \\
\hline I 4079 & I I 57 & I 8846 & I 233 & $25^{\circ} 5^{8}$ & I 158 & 27431 & 945 \\
\hline 14094 & I I I I & I9004 & 307 & 25067 & 413 & 27458 & I $45^{8}$ \\
\hline I 4344 & I 206 & 19027 & 535 & 25134 & 648 & 27498 a & 1517 \\
\hline $1455^{2}$ & 227 & 19055 & 628 & 25138 & 1058 & 27515 & 873 \\
\hline $14930 \mathrm{a}$ & 1362 & 19075 & 1546 & 25175 & I 505 & 27593 & 402 \\
\hline 15048 & I095 & 19295 & 575 & $2525^{\circ}$ & I 47 & 27774 & 76 \\
\hline I 5099 & 776 & I930I & I38 & 25272 & 190 & $2784^{\circ}$ & 903 \\
\hline 15267 & 1042 & I94I9 & I 326 & $25285 b$ & 623 & 27895 & 1023 \\
\hline 15282 & 297 & 19547 & 1260 & $2534^{\circ}$ & 975 & 27977 & IOI 7 \\
\hline 15310 & 802 & I9596 & 1475 & 25359 & 1305 & 28044 & 1357 \\
\hline 15316 & I 533 & 19645 & 1142 & 25444 & 1401 & $2805^{2}$ & 1002 \\
\hline I $5450 \mathrm{~b}$ & 868 & 19668 & x 208 & 25489 & I 518 & 28062 & I66 \\
\hline 15471 & 982 & I9688 & 75 & 25540 & I 107 & 28079 & 768 \\
\hline 15477 & I 201 & I9805 & 1197 & $255^{8} 3$ & 400 & 28117 & 1078 \\
\hline I 5546 & I I 80 & 19872 & I IO & 25667 & 676 & 28273 & 300 \\
\hline I 5549 & 1477 & I 9873 & 439 & 25668 & I 288 & 28286 & 964 \\
\hline I 5558 & 96 & I 9883 & 602 & 25696 & $8 \mathrm{I}$ & 28298 & I I 43 \\
\hline I 5628 & $145 \mathrm{I}$ & I99 I 5 & 1005 & 25707 & 594 & $2834 \mathrm{I}$ & 897 \\
\hline I $5652 / 3$ & 1378 & 20029 & 645 & 25714 & 66 & 28394 & I 3 \\
\hline 15860 & 379 & 20217 & 312 & $2574 \mathrm{I}$ & 45 & $2844^{8}$ & IOI \\
\hline I 5921 & 288 & 20294 & 870 & $2577 \mathrm{I}$ & 1167 & 285 I 2 & 64 \\
\hline 16483 & I 169 & $2044^{8}$ & 1304 & 25812 & $125^{\circ}$ & 28598 & 1508 \\
\hline 16621 & 869 & 206 I I & 397 & 25833 & 823 & 28661 & 1317 \\
\hline 66653 & 1290 & 20726 & 1056 & $25854 \mathrm{~b}$ & 483 & 28695 & 707 \\
\hline 16803 & 1488 & 20909 & $60_{4}$ & $2586 \mathrm{I}$ & 265 & 28734 & 933 \\
\hline
\end{tabular}




\begin{tabular}{|c|c|c|c|c|c|c|c|}
\hline CIL VI & & CIL VI & & CIL V & & CIL V & \\
\hline 28759 & I 240 & 32772 & 1036 & $3^{87}$ & 488 & 4157 & 489 \\
\hline 28770 & 266 & 32783 & 1298 & 410 & 201 & 4293 & I IO4 \\
\hline 28778 & 1425 & 32808 & 1205 & 466 & $4^{20}$ & 4373 & 1212 \\
\hline 28807 & 1271 & 32965 & 209 & 483 & 713 & 4391 & I 45 \\
\hline $2884^{\circ}$ & 82 & $333^{89} a$ & 462 & 533 & 355 & $455 \mathrm{I}$ & I 44 \\
\hline $2888 I$ & I I 33 & 33500 & 1452 & 688 & $4 I$ & 4554 & \\
\hline 28910 & 404 & 33602 & 332 & 828 & 1566 & 4573 & 65 \\
\hline 28916 & 262 & 33634 & 169 & 1040 & I I 59 & 4669 & 1045 \\
\hline 28999 & 670 & 33814 & I5 I I & I 878 & IOI 2 & 5030 & 1008 \\
\hline 29085 & I 249 & 33833 & 391 & 2088 & 283 & $5036 \mathrm{c}$ & $55^{6}$ \\
\hline 29195 & 1072 & 33864 & 703 & 2 I 82 & I 445 & 5370 & IO \\
\hline 29235 & 1390 & 33899 & 850 & 2210 & 222 & 5418 & \\
\hline 29264 & 220 & 33907 & 569 & 2297 & 276 & $544^{6}$ & 36 \\
\hline 29403 & I 476 & 33911 & 213 & 2631 & I I 78 & 5502 & \\
\hline 29544 & 1130 & 33981 & 454 & 2640 & I $47 \mathrm{I}$ & 5667 & IO2I \\
\hline 29579 & 1500 & 34025 & 1217 & 2782 & I 398 & 5784 & 1307 \\
\hline 29680 & 771 & 34026 & IOIO & 2833 & 215 & 5798 & I 374 \\
\hline $29849 a$ & 1080 & 34060 & 1025 & 2848 & I 440 & 6023 & I $48 \mathrm{I}$ \\
\hline 29884 & 426 & 34124 & I IOI & 2894 & 760 & 6243 & 38 \\
\hline 29890 & 603 & 34635 a & 301 & 2913 & 122 & 6622 & 47 \\
\hline 29924 & 47 & 34639 & 9 & 2988 & 448 & 6970 & 1248 \\
\hline 29925 & 636 & 34676 & 221 & 3028 & 701 & 7156 & 1375 \\
\hline 29945 & I 68 & 35118 & $5^{\circ}$ & 3131 & I06 & 7271 & I 38 \\
\hline 29947 & I I 68 & 35279 & 1292 & 3147 & I 346 & 7384 & 59 \\
\hline 29958 & I 296 & 35381 & 1057 & 3157 & I 269 & 7413 & \\
\hline 29983 & I 255 & 35434 & 874 & 3198 & $23^{6}$ & 7467 & I 43 \\
\hline 30073 & 268 & 35454 & 875 & 3302 & II 32 & 7505 & $\mathrm{IO}_{4}$ \\
\hline 30 I I I & 148 & 35547 & 1033 & 3319 & IOI5 & 7517 & I 447 \\
\hline 30118 & 1402 & $35548 \mathrm{a}$ & 1146 & 3327 & 943 & 7819 & 98 \\
\hline 30123 & $67 \mathbf{I}$ & 35868 & $124 \mathrm{I}$ & 3349 & 685 & 7928 & 1059 \\
\hline 30125 & $34 \mathrm{I}$ & 35915 & I 544 & 3357 & 94 & 7947 & 502 \\
\hline 30510 & 1308 & 35987 & 1287 & 3455 & 1347 & 7998 & 63 \\
\hline 30758 & 1554 & 36377 & 182 & 3485 & 216 & 8037 & $6 I$ \\
\hline 30865 & 638 & 36653 & $115 I$ & 3520 & 107 & 8247 & 66 \\
\hline 30894 & 1483 & CIL VII & & 3521 & 562 & 8424 & 54 \\
\hline 31007 & 1182 & 66 & $5 \mathrm{I} 4$ & 3577 & 460 & 8490 & 155 \\
\hline 31009 & 98 & 80 & I 156 & 3582 & I 530 & 8530 & 154 \\
\hline 31038 & 1070 & 133 & 836 & 3596 & 353 & 8573 & I 16 \\
\hline 31066 & 229 & 140 & 455 & 3611 & 566 & 8732 & 152 \\
\hline 31169 & 388 & 265 & 1376 & 3617 & 828 & 8789 & I I 54 \\
\hline $3185^{\circ}$ & 1064 & 355 & 826 & 3716 & 1336 & 88 I 2 & \\
\hline 31852 & 1202 & 631 a & 329 & 3741 & 1403 & 9020 & I 25 \\
\hline $32398 a$ & 1553 & 972 & 445 & 3762 & 983 & 9056 & 19 \\
\hline 32547 & 1466 & 1074 & 1049 & 3783 & I 433 & $905^{8 b}$ & I 26 \\
\hline 32565 & I 65 & 1336,411 & 843 & 3899 & 267 & 9069 & I 2 ] \\
\hline 32573 & 677 & $1336,43^{8}$ & 519 & 3933 & I 246 & 9072 & I337 \\
\hline 32583 & 396 & 1336,605 & 788 & $3936 / 7$ & 309 & $908 \mathrm{I}$ & 34 \\
\hline 32589 & II I I & I336, I I90a & 364 & 4009 & I9I & $9 \Upsilon 14$ & 55 \\
\hline 32596 & 1170 & CIL VIII & & 4105 & 395 & 9127 & 1360 \\
\hline 32647 & $55^{1}$ & $132 \mathrm{a}$ & I 448 & $4 I I I$ & $95^{2}$ & $9139 b$ & $54^{8}$ \\
\hline 32694 & 217 & 152 & 630 & 4120 & I I 49 & $9156 a$ & 1435 \\
\hline 32744 & 394 & 292 & 1484 & $4120 \mathrm{~b}$ & 1037 & 9I $8 \mathrm{I}$ & 67 \\
\hline 32762 & 528 & 371 & 1455 & 4122 & 521 & 9189 & $26 c$ \\
\hline
\end{tabular}




\begin{tabular}{|c|c|c|c|c|c|c|c|}
\hline CIL VIII & & CIL VII] & & CIL IX & & CIL IX & \\
\hline 9200 & 345 & I9I89c & 136 & 1055 & 765 & 4418 & 359 \\
\hline 9273 & 912 & I9606 & 282 & 1204 & 989 & 4805 & 944 \\
\hline 9347 & 908 & I9929 & $3^{8}$ & I 209 & 6 & 4822 & 1313. 683 \\
\hline $93^{8 I}$ & I 266 & 20062 & 756 & 1276 & 142 & 5037 & $95^{6}$ \\
\hline 9426 & 92 & 20499 & I 524 & 1287 & 697 & 5325 & $93^{\circ}$ \\
\hline $943^{\circ}$ & 913 & 20505 & 457 & 1315 & 739 & 5392 & 449 \\
\hline $943^{2}$ & 3 II & 20549 & 840 & 1424 & I $43^{8}$ & 5417 & I 479 \\
\hline $9442 \mathrm{a}$ & $35^{\circ}$ & 20686 & 37 & 1481 & 510 & 5577 & $3^{86}$ \\
\hline 9462 & 984 & 20734 & 40 & I 498 & 434 & 5813 & 493 \\
\hline 9493 & $933^{a}$ & 20786 & 544 & 1515 & 809 & 5860 & I397 \\
\hline 9638 & 342 & $20855 a$ & 337 & I 524 & 1139 & 5908 & 22 \\
\hline 9768 & 343 & 20945 & 1266 & 1681 & I $562 a$ & 6081 & $45^{\circ}$ \\
\hline 9910 & 546 & 21014 & $145^{\circ}$ & 1826 & $155^{\circ}$ & CIL $\mathrm{X}$ & \\
\hline 9985 & $56 I$ & $2 \mathrm{IO} 3 \mathrm{I}$ & I 395 & I $93^{8}$ & 1244 & I 74 & $155 \mathrm{I}$ \\
\hline 9998 & 1472 & 21038 & 663 & 2 II I & $80 I$ & 216 & 444 \\
\hline 10475,22 & 436 & 21068 & 1329 & 2123 & 375 & 270 & $95^{\circ}$ \\
\hline 10575 & 1085 & 2 I086 & 891 & 2 I 5 I a & 1152 & 380 & 995 \\
\hline 10982 & 587 & 21087 & 44 & 2164 & 1507 & 423 & 292 \\
\hline I 1306 & 27 & 2 III 6 & 749 & 2302 & $\begin{array}{l}1 \\
1\end{array}$ & 477 & I 557 \\
\hline I I 594 & 1372 & $2 \times 145$ & 791 & 2305 & 807 & 623 & 1067 \\
\hline 12159 & I 2 I 4 & 21204 & $25 \mathrm{I}$ & 2398 & $9 \times 1$ & 633 & I I 48 \\
\hline 12504 & 859 & 21214 & 664 & 2416 & 967 & 649 & 1462 \\
\hline $12505 \mathrm{~b}$ & 860 & 2 I 24 I & 418 & 2450 & 274 & 719 & 876 \\
\hline $1264^{\circ}$ & I 365 & 21284 & 192 & 2492 & I 454 & I2 I I & 532 \\
\hline I 2657 & I3I 8 & 21345 & 1328 & 2717 & 734 & I3O7 & 331 \\
\hline I 2745 & $\mathrm{r}_{47} \mathrm{O}$ & 2148 I a & I IOO & 2760 & $74^{\circ}$ & 1323 & 805 \\
\hline 12794 & 1087 & 21489 & 675 & 2878 & I 123 & 1692 & $155^{8}$ \\
\hline $1293^{\circ}$ & I 124 & 21531 & 1034 & 2880 & 347 & 1741 & 539 \\
\hline I 2943 & 322 & 21564 & 1007 & 2892 & 751 & 1760 & 1520 \\
\hline I3I 34 & 662 & 2 I 569, I & 549 & 2893 & 508 & 1775 & 419 \\
\hline 14420 & 596 & 21644 & 665 & $299 I$ & 710 & 1974 & 372 \\
\hline 14601 & 554 & 21810 & 1052 & $304^{\circ}$ & 417 & 2056 & I309 \\
\hline 14603 & 1096 & 22566 & $54^{2}$ & 3058 & 494 & 2066 & 1277 \\
\hline 14683 & 1567 & CIL IX & & 3093 & 896 & 2070 & 290 \\
\hline 15147 & 285 & IO4 & 779 & $3 \mathrm{IO}_{3}$ & 906 & 2079 & $83^{I}$ \\
\hline I 5 I 49 & I 264 & 140 & 772 & 3105 & 1429 & 2145 & 877 \\
\hline I 59 I5 & I 2 I 3 & 154 & 487 & 3271 & 57 & 2147 & 990 \\
\hline 16208 & $5^{63}$ & 156 & 741 & 3337 & 654 & 2 I 54 & 730 \\
\hline I63I3a & $8_{4} I$ & I75 & 672 & $33^{87} a$ & I 430 & 2184 & 536 \\
\hline 16566 & I 413 & I 79 & 433 & $344^{8}$ & 696 & 2224 & 14122 \\
\hline I $658 \mathrm{I}$ & 892 & 197 & 900 & 3472 & I 127 & 2244 & 424 \\
\hline 16752 & I 495 & 229 & 149 & 3473 & I I9 & 2280 & 77 \\
\hline $17014 b^{2}$ & 799 & 249 & 199 & 3763 & 780 & 2289 & 1030 \\
\hline 17402 & 1366 & 369 & 755 & $3^{821}$ & 634 & 2333 & $65^{2}$ \\
\hline 17422 & 557 & 384 & 55 & 3868 & $45^{2}$ & $2354 \mathrm{~b}$ & 463 \\
\hline I 7497 & 1086 & 385 & 202 & 4028 & 679 & 2383 & 259 \\
\hline 18199 & 1433 & 820 & 1089 & 4032 & 7 & 2394 & 898 \\
\hline 18224 & 555 & 871 & 684 & 4088 & 762 & 2466 & 178 \\
\hline 18419 & 490 & 896 & 808 & 4123 & 363 & 2487 & 1035 \\
\hline $1845^{\circ}$ & 185 & 959 & 1428 & 4175 & 968 & 2496 & 1211 \\
\hline I 8792 & 1247 & 984 & 923 & 4230 & 260 & $254^{8}$ & 533 \\
\hline 19146 & I 408 & 998 & 309 & 4326 & 541 & 2559 & 174 \\
\hline 19174 & I I 8I & 1023 & $5^{6}$ & 44 I 6 & 326 & 2598 & 18 \\
\hline
\end{tabular}


v inhaltsverzeichnis

\begin{tabular}{|c|c|c|c|c|c|c|c|}
\hline $\begin{array}{l}\text { CIL X } \\
2622\end{array}$ & I IO9 & $\begin{array}{l}\text { CIL X } \\
8044,1\end{array}$ & 272 & $\underset{2 I_{3}}{\text { CII } X I}$ & 830 & $\begin{array}{l}\text { CIL XII } \\
3925\end{array}$ & 847 \\
\hline 2645 & 1 216 & 8072,1 & 1406 & 230 & 336 & 4159 & 157 \\
\hline 2728 & 688 & 8119 & 432 & 319 & 99 & $4247 b$ & 829 \\
\hline 2729 & I364 & 8189 & 214 & 345 & 1417 & 4355 & 43 \\
\hline 2792 & I 301 & $82 I 4$ & 854 & 394 & 328 & 4399 & 1027 \\
\hline 2801 & 208 & 8249 & 852 & 460 & 464 & 4487 & 822 \\
\hline 2822 & 962 & 8364 & 1444 & 678 & 735 & 4495 & I97 \\
\hline 2933 & 1426 & 8402 & I I 74 & $682 a$ & 1361 & 4514 & I 528 \\
\hline 2965 & 980 & CII XI & & 704 & I I 2 & 4517 & 708 \\
\hline 2993 & 824 & 84 & 957 & 743 & 627 & 4833 & 763 \\
\hline 3010 & 1185 & 137 & I 493 & 746 & 320 & 4907 & 947 \\
\hline 3032 & 1083 & 147 & 1548 & 794 & 845 & 4979 & 963 \\
\hline 3148 & $28 \mathrm{I}$ & 216 & 286 & 807 & 928 & 5019 & 398 \\
\hline 3160 & 1300 & 466 & 629 & 874 & $6 I_{4}$ & 5193 & 825 \\
\hline 3344 & I343 & $5^{16}$ & 1526 & 915 & 373 & $5^{2} 43$ & 716 \\
\hline 3395 & 376 & 619 & I 280 & 923 & 810 & 5457 & 59 \\
\hline 3503 & 53 & 746 & II 45 & 987 & $I_{4} 10$ & 5502 & 31 \\
\hline 3659 & 1383 & 932 & 7 I I & I 109 & I 6I & 5517 & 23 \\
\hline 3824 & 853 & 947 & $24 I$ & I I I 2 & 761 & 5519 & 318 \\
\hline 3947 & 690 & 987 & I 210 & I I 28 & 383 & 5561 & I6 \\
\hline 4102 & 939 & IOI 6 & I75 & I 162 & 591 & 5682,43 & $44^{6}$ \\
\hline $4 \simeq 13$ & 806 & I IO3 & I I 44 & I I 93 & 150 & 5685,18 & 786 \\
\hline 4183 & $43 I$ & I4I I & 84 & I 2 IO & I7 & 5686,356 & 615 \\
\hline $4292 \mathrm{a}$ & 104 & I 491 & 655 & I $25^{\circ}$ & 93 & $5686,734 d$ & 737 \\
\hline $43^{88}$ & 884 & 1616 & 1359 & I 339 & 1215 & 5686, I 2 I I & 590 \\
\hline 4399 & 955 & 2564 & I 134 & I 356 & $\mathrm{I} 43$ & 5687,9 & 656 \\
\hline 4407 & I I 55 & 2657 & 252 & I 381 & 1218 & 5698,3 & 333 \\
\hline $443^{I}$ & 1020 & 2720 & IOI I & 1416 & $4^{85}$ & 5698,6 & 887 \\
\hline 4915 & 54 & 3266 & I I I 4 & 1466 & I $546 a$ & 5827 & 754 \\
\hline 4936 & 246 & 3522 & 1431 & 1477 & I 56 & CII XIII & \\
\hline 5336 & I333 & 3539 & 2 & 1728 & 171 & $\mathrm{I} 60$ & I I 20 \\
\hline 5349 & 1559 & 3541 & I 83 & 1751 & 505 & 510 & $83^{8}$ \\
\hline 5371 & 633 & 3939 & $35 \mathrm{I}$ & 1753 & IIOZ & $633 b$ & 1003 \\
\hline 5434 & 1136 & 4010 & 635 & 1814 & $33^{8}$ & 715 & 722 \\
\hline $55^{89}$ & 1485 & $444^{6}$ & 1370 & 1939 & I 522 & 816 & 787 \\
\hline 5815 & I 273 & 5265 & I 565 & I957 a & $68 I$ & $\mathrm{IO}_{4} \mathrm{I}$ & 12 \\
\hline 5939 & I 137 & 5749 & 1563 & I97 I & 2 I I & I I 96 & 1527 \\
\hline 5980 & 978 & 5779 & I 147 & 2016 & 523 & 1839 & 179 \\
\hline 6608 & 878 & 5784 & 782 & 2037 & 800 & I 981 & 522 \\
\hline 6565 & I 560 & 5796 & 242 & 2366 & 203 & 2000 & 819 \\
\hline 6656 & $156 \mathrm{I}$ & 5865 & I I 19 & 2426 & 718 & 2015 & $98 \mathrm{I}$ \\
\hline 6785 & I 274 & 5939 & I 299 & 2674 & 1486 & 2016 & 1531 \\
\hline 6977 & I 177 & 5996 & I 564 & 2762 & 415 & 2087 & $13^{8} 5^{a}$ \\
\hline 7042 & 1236 & 6078 & 1060 & 2819 & 1334 & 2103 & $137 \mathrm{I}$ \\
\hline 7545 & 726 & 6080 & I 373 & 2862 & 929 & 2237 & 1414 \\
\hline $7586 a$ & 139 & 6417 & I 345 & 3349 & 500 & 2457 & 1046 \\
\hline 7596 & 1478 & 6727 & 834 & 3361 & 1418 & 2515 & 356 \\
\hline 7648 & 1084 & $673^{\circ}, 4$ & 1187 & 3405 & 1377 & $253 I$ & 24 \\
\hline 7668 & 732 & CIL XII & & 3453 & I 5 I 5 & $254^{8}$ & 60 \\
\hline 7692 & 832 & I & 626 & 3473 & I I I 5 & 2646 & 733 \\
\hline 7808 & 709 & 22 & I 536 & 3523 & I72 & 2666 & I I 5 \\
\hline $7809 b$ & 653 & I 22 & 744 & 3573 & 362 & 3033 & 299 \\
\hline 7947 & 905 & I94 & 1512 & 3694 & 306 & 3456 & $45^{8}$ \\
\hline
\end{tabular}




\begin{tabular}{|c|c|c|c|c|c|c|c|}
\hline CIL XIII & & CII & & CIL XIV & & $\mathrm{CE}$ & \\
\hline $345^{8}$ & 87 & 904 & $\operatorname{II} 98$ & 2780 & 233 & 80 & 920 \\
\hline 3638 & 1047 & 914 & 429 & $284 \mathrm{I}$ & 205 & IOI & 1381 \\
\hline 3983 & I94 & $95^{\circ}$ & 335 & 2871 & 1387 & I I 2 & 1086 \\
\hline 4166 & 714 & 966 & I 529 & 2917 & 578 & I 18 & 633 \\
\hline 4532 & 163 & 1009 & I 160 & 2934 & I $55^{6}$ & I 20 & 635 \\
\hline 5270 & $74^{8}$ & 1033 & 177 & 3313 & 354 & I 28 & $1 \pm 64$ \\
\hline 5554 & I 436 & $105 \mathrm{I}$ & $99 \mathrm{I}$ & 3323 & 407 & 133 & I I 49 \\
\hline 5697 & 25 & 1136 & $93^{8}$ & 3344 & I 525 & I 4 I & 1008 \\
\hline 6460 & $8 I I$ & 1137 & 543 & 3478 & 966 & 142 & 499 \\
\hline 7119 & 613 & 1151 & 406 & 3507 & 466 & 170 & I 494 \\
\hline 7441 & 475 & 1153 & 1382 & 3525 & I 4 I 5 & 178 & 55 \\
\hline $7516 a$ & I 335 & I 169 & 498 & 3571 & $45^{6}$ & I 86 & I 9 \\
\hline 7521 & $135^{2}$ & I 170 & 742 & 3630 & 743 & 189 & 428 \\
\hline $755^{\circ}$ & 857 & I 195 & II 8 & 3831 & 919 & 192 & II 5 I \\
\hline 7554 & 858 & 1198 & 988 & 4010 & 1225 & 198 & I5OI \\
\hline 7709 & I 281 & 1324 & 223 & 4015 & I009 & 199 & 998 \\
\hline 7753 & 125 I & 1342 & I I I 8 & 4231 & 302 & 201 & I 49 I \\
\hline 7865 & I 54 I & 1347 & 368 & CIL XV & & 222 & $43 I$ \\
\hline 8274 & 1324 & 1357 & $134^{2}$ & 635 & 1232 & $24 I$ & 634 \\
\hline 8364 & 974 & 1370 & 904 & $650 a$ & 798 & 280 & 437 \\
\hline 8384 & 240 & I 385 & 959 & 2177 & 1000 & 339 & 637 \\
\hline 8874 & 1032 & I4OI & 926 & 4653 & 244 & 340 & 752 \\
\hline $\mathrm{CIL}^{+} \mathrm{XIV}$ & & I 463 & 705 & 4855 & 427 & 342 & 656 \\
\hline 8 & 1332 & 1473 & I 235 & 5929 & I 23 I & 405 & $155^{I}$ \\
\hline 25 & 470 & I 474 & 960 & 7036 & 1416 & 454 & 627 \\
\hline 36 & 576 & 1527 & 330 & 7172 & 649 & 470 & 373 \\
\hline 39 & I35 I & 1574 & 49 & 7173 & 650 & 474 & 1205 \\
\hline 62 & 618 & $166 I$ & I 506 & 7176 & 1183 & 475 & 643 \\
\hline 64 & 408 & I 688 & 520 & 7181 & 1315 & 476 & 642 \\
\hline 107 & 83 & 1726 & 619 & 7183 & 1175 & 479 & I395 \\
\hline $15^{8 / 9}$ & I 459 & I 731 & 920 & 7919 & $55^{8}$ & 490 & 341 \\
\hline 166 & 1502 & I755 & 1184 & $7199 a$ & 1256 & 493 & I 180 \\
\hline I97 & 927 & 1784 & 954 & 7211 & 1238 & 495 & 628 \\
\hline 238 & 52 & I793 & 117 & Eph. epigr. & VIIĬ & 496 & 1024 \\
\hline 308 & 965 & I 821 & 1405 & $6 \mathrm{x}$ & $78 \mathrm{I}$ & 497 & $I_{4} 22$ \\
\hline 429 & 689 & 1 868 & IOI 4 & 78 & 512 & 505 & 629 \\
\hline 487 & 695 & I 873 & I 164 & 126 & I494 & 509 & 18 \\
\hline 497 & 1098 & 1874 & 428 & 152 & I I 40 & 512 & I 375 \\
\hline 523 & 273 & 2080 & 430 & 160 & 68 & 516 & 630 \\
\hline 525 & 778 & 2082 & 738 & 166 & I 29 & $5 \times 7$ & 1360 \\
\hline 526 & 1105 & 2120 & I006 & 238 & I 125 & $54^{8}$ & 1025 \\
\hline 535 & 804 & 2165 & I 555 & 257 & 1108 & 549 & 1290 \\
\hline 553 & 1492 & 2170 & 992 & 316 & 162 & 563 & 1405 \\
\hline 584 & 694 & 2286 & I I 4 & 385 & 534 & 578 & $3^{81}$ \\
\hline 595 & IO3 & 2325 & 547 & 426 & I39 & 593 & 344 \\
\hline 614 & $5 I$ & 2341 & 973 & 430 & 885 & 595 & IOI3 \\
\hline 637 & 678 & 2346 & $2 I$ & 504 & 360 & 597 & 1283 \\
\hline 671 & 1467 & 2379 & $44^{2}$ & 529 & 855 & 600 & 671 \\
\hline 688 & 977 & 2555 & 1243 & 798 & I396 & 604 & 1169 \\
\hline 766 & 1063 & 2653 & 1001 & I 4 p. 360 & 479 & 613 & 1211 \\
\hline 783 & $35^{8}$ & 2656 & 846 & I 5 p. 360 & 71 & 626 & I 449 \\
\hline 836 & 579 & 2710 & 127 & 128 p. 408 & 207 & 635 & 136 \\
\hline 849 & I 196 & 2772 & 1369 & 246 p. 476 & 369 & 637 & 325 \\
\hline
\end{tabular}




\begin{tabular}{|c|c|c|}
\hline $\mathrm{CE}$ & & CE \\
\hline 642 & 710 & I 826 \\
\hline 646 & 1407 & 1851 \\
\hline 647 & 632 & $\mathrm{De}$ \\
\hline $90 \mathbf{I}$ & 176 & 705 \\
\hline 923 & 792 & 792 \\
\hline 1043 & $14^{6} 9$ & I 283 \\
\hline 1094 & 148 & I 576 \\
\hline I 145 & 707 & 1709 \\
\hline I I 50 & 1097 & $173^{6}$ \\
\hline I I 73 & I 166 & 1741 \\
\hline I I 89 & I 274 & I 744 \\
\hline I I 90 & I359 & I 798 \\
\hline 1216 & I 388 & 1823 \\
\hline 1253 & I 106 & 1980 \\
\hline 1265 & 654 & 2045 \\
\hline 1267 & 1320 & 2047 \\
\hline 1308 & I I 48 & 2055 \\
\hline 1318 & 429 & 2089 \\
\hline 1319 & 54 & 2110 \\
\hline 1324 & I 216 & 2147 \\
\hline 1328 & 1372 & 2207 \\
\hline 1331 & 1275 & 2305 \\
\hline 1332 & I 4 I 3 & 2307 \\
\hline 1463 & 1468 & 2328 \\
\hline 1474 & $36 I$ & 2332 \\
\hline $154 \mathrm{I}$ & 1402 & 2344 \\
\hline 1580 & I 493 & 2346 \\
\hline 1581 & 631 & 2349 \\
\hline 1582 & $1387 a$ & 2415 \\
\hline 1583 & I 5 I I & 2467 \\
\hline 1588 & 1319 & 2568 \\
\hline I 593 & 1371 & 2763 \\
\hline 1599 & 1427 & 2783 \\
\hline 1601 & 660 & 2784 \\
\hline I 606 & 662 & 2791 \\
\hline 1607 & 282 & 2843 \\
\hline 1608 & IOI 5 & 2883 \\
\hline 1788 & I 247 & 3051 \\
\hline I799 & 168 & 4316 \\
\hline 1822 & 242 & 5632 \\
\hline 1823 & I373 & 5702 \\
\hline
\end{tabular}

\begin{tabular}{|c|c|c|c|c|}
\hline & $\mathrm{De}$ & & $\mathrm{De}$ & \\
\hline $28 I$ & 5713 & 1566 & 8223 & 1382 \\
\hline 614 & 5902 & 1343 & 8225 & 423 \\
\hline & 6001 & 1369 & 8228 & IоI9 \\
\hline 1565 & 6 I I 4 & 1557 & 8234 & I397 \\
\hline $155^{8}$ & 6186 & 430 & 8237 & 923 \\
\hline 1555 & 6199 & 1006 & 8249 & $4^{80}$ \\
\hline 393 & 6824 & I 567 & 8266 & 1005 \\
\hline IOI 2 & 7221 & 1563 & 8286 & I I 94 \\
\hline 465 & 7300 & IOI I & 8299 & 273 \\
\hline 167 & 7518 & 1026 & 8304 & 379 \\
\hline 1318 & 7589 & 1097 & 8334 & 250 \\
\hline 1207 & 7648 & 8 I9 & 8335 & 36 \\
\hline 797 & 7674 & 1272 & 8353 & 837 \\
\hline I 493 & 7734 & 633 & 8370 & 1262 \\
\hline I 465 & 7735 & 391 & 8375 & $155^{6}$ \\
\hline 444 & $774 \mathrm{I}$ & $138 \mathrm{I}$ & 8380 & I 553 \\
\hline I $4 \mathrm{I}$ & 7893 & $125^{8}$ & 8382 & I 230 \\
\hline $55^{\mathrm{I}}$ & 7922 & IOI 4 & 8383 & I0I8 \\
\hline I $35^{8}$ & 7927 & 1305 & 8384 & 575 \\
\hline 1088 & 7965 & 1306 & 8392 & I 287 \\
\hline 1298 & 7990 & 1422 & 8447 & I374 \\
\hline 1096 & 8080 & 1277 & 8455 & 1008 \\
\hline I I 22 & 8089 & $40 I$ & $845^{8}$ & $155^{\circ}$ \\
\hline 760 & 8090 & 407 & $846 I$ & 655 \\
\hline 86 & 8100 & 42 & 8465 & 266 \\
\hline 448 & $8 \mathrm{IO}_{2}$ & 1181 & 8495 & I087 \\
\hline 759 & 8 I 6 & I 235 & 8496 & I I I I \\
\hline 1251 & 8127 & 942 & 8500 & 665 \\
\hline 555 & 8129 & IOO & $85 \div 5$ & 1094 \\
\hline I37 & 8 I $32^{2}$ & I I $4^{8}$ & 8526 & 1426 \\
\hline 663 & 8134 & I 164 & $855^{8}$ & I 535 \\
\hline I $26 \overline{6}$ & 8 I37 & 1303 & $855^{8} a$ & 1539 \\
\hline 1040 & 8145 & 1480 & 8727 & 649 \\
\hline 1324 & 8146 & 265 & $875^{\circ}$ & 850 \\
\hline 209 & 8151 & 1408 & $875 \mathrm{r}$ & 851 \\
\hline 1036 & $816 \mathrm{Ib}$ & 291 & 8752 & 856 \\
\hline 376 & 8182 & 168 & 8753 & $86 \mathrm{I}$ \\
\hline 777 & 8186 & I I 29 & 8754 & 859 \\
\hline I 554 & $8187 a$ & I I 68 & 8756 & 862 \\
\hline 1560 & 82 I I & I 439 & 8757 & 863 \\
\hline 156 I & 8212 & 63 & & \\
\hline
\end{tabular}

\section{VERBESSERUNGEN}

197 anm. 3 : : oder o(biit), o(bitae)

423 lies: (sestertium)

545 lies: (nach add. p. 970): bene laves: oze a(ssem) des, cras gratis: res tuta. salvu lave(s) e(t) bono (eius), qi fieri iusit ex s/uo parcimonio ?]. vgl. CIL V 4500 und CIL a. a. o.

981 lies: Marinius 1037 anm. 'vgl. nro II 49 ' 